\title{
Research article \\ Stress level among the final year medical students at an urban medical college: A cross-sectional study
}

\author{
Monika Battula ${ }^{1}$, Preethi Arunashekar ${ }^{1}$, Arockia John' ${ }^{1}$, Radha ThiyagaRajan ${ }^{1}$, P. N. Vinoth ${ }^{2}$ \\ ${ }^{1}$ Junior Resident, ${ }^{2}$ Associate Professor, Department of Pediatrics, Sri Ramachandra Institute of Higher Education and Research, \\ Porur, Chennai 600 116, Tamil Nadu, India
}

(Received: October $2020 \quad$ Revised: December $2020 \quad$ Accepted: January 2021)

Corresponding author: P. N. Vinoth. Email: Vindoc1977@gmail.com

\begin{abstract}
Introduction and Aim: Stress is a condition, which is perceived through environmental threats and events and is initiated by stressors. Globally there is a high prevalence of stress among the medical students which impacts the cognitive and learning ability of medical students and also their mental wellbeing. The present study was conducted to identify the stressors and measure the intensity of the stress among final year medical students at Anurban Medical College in Chennai.

Methods: This cross-sectional study was conducted among 250 final year undergraduate medical students between July and September 2018. The students were exposed to medical student stressor questionnaire (MSSQ-20), consisting of six domains including academic related stressors (ARS), teaching related stressors (TLRS), intrapersonal and interpersonal development stressors (IRS), social related stressors (SRS), desire related stressors (DRS) and group activities related stressors (GARS). The stress levels were graded as mild, moderate, high and severe.
\end{abstract}

Results: Majority of the participants were females (56\%) and the mean age was $22.17 \pm 0.94$ years. MSSQ-20 analysis showed that $138(55 \%)$ students perceived severe stress due to ARS and 85(34\%) students were prone to high stress as a result of GARS. The mean score was higher for ARS, followed by GARS and the least score was observed in DRS.

Conclusion: Since academics and group activities are the most dominant stressors, there is a need for restructuring the medical education delivery system to enable the medical students acquires knowledge without compromising their health and wellbeing.

Keywords: Stress; medical students; MSSQ-20; academic related stress.

\section{INTRODUCTION}

$\mathrm{M}$ edical education is a highly demanding and competitive professional course globally and it has significant impact on the mental and physical well-being of medical students. An array of factors such as fear towards exams, high expectations from parents, curricula pressure, less leisure time, financial constraints and preparation for further specialization leads to stress among the undergraduate medical students $(1,2)$. Albeit stress may alter the academic performance and can lead to depression, burnout and somatic complaints among the medial students (3).

The incidence of psychological stress is higher among the medical students as compared to the other non-medical students. (4). A meta-analysis study encompassing 195 studies from 47 countries, involving 129,000 medical students shows that $27.2 \%$ and $11.1 \%$ displayed depression symptoms and suicidal behaviour respectively (5). Among the various factors, academic problems are the mainstay in the cause of stress among the medical students. The chronic stress also related to overloaded curriculum and repeated examinations. In addition, the medical college environment is highly restrictive and competitive, further imposing additional psychological burden among the medical students (6, 7).

The stress among medical students starts during their first year of academics and remains till the end of the study period. Rotenstein et al., highlighted that the stress level is constant throughout the medical course period (5). Abdulghani et al. reported that medical students displayed elevated stress level during the first year of course with a concomitant decrease in advancing years, but not in the final year of the study course (8). Mounting reports, delineates that there is a marked increase in the stress level among the medical students with progressing study years $(9,10)$. With this backdrop, the present study was undertaken to identify the stressors and measure the intensity of the stress caused by the stressors among final year M.B.B.S students at Anurban Medical College.

\section{METHODS}

The present descriptive cross-sectional study was conducted among the final year medical students of the present tertiary teaching institution between July and September 2018. Institutional Ethics Committee 
approval was obtained before the conduction of study. The study purpose was clearly explained to the participants and the written informed consent was obtained from each participant prior to the data collection. Sample size was calculated based on the study conducted by Gupta et al. where the prevalence of stress was found to be $91.2 \%$ (1). At $95 \%$ confidence limits and $4 \%$ relative precision, the sample size was calculated as 231 . Accounting $10 \%$ for non-response, the sample size was further increased to 254 and rounded off to 250 .

\section{Data collection tools}

A structured, self-administered questionnaire was used to obtain information regarding the demographic profiles of the participants. Medical Students Stressor Questionnaire (MSSQ)was used to obtain data regarding the sources of stress (11). The MSSQ consists of 20 (MSSQ-20) events which was pre-designed, pre-tested, semi-structured and validated for evaluating the stressors and severity of stress perceived by medical students.

The questions in the MSSQ-20 were addressed to six domains of stress which included, academic related stressors (ARS), which involves tests/examinations, falling behind in reading schedule, huge pending content to be learned, lack of time to review what has been learnt, heavy workload, teaching related stressors (TLRS), involves not enough feedback from teachers, inadequate study material, lack of guidance, teaching skills, intrapersonal and interpersonal development stressors (IRS) involves verbal or physical abuse by students, teachers and personnel and conflict with teachers, social related stressors (SRS) involves unable to answer questions from patients, talking to patients about personal problems, facing illness or death of patients, desire related stressors (DRS) involves unwillingness to study medicine, parental wish to study medicine, and group activities related stressors (GARS) involves participation in class presentation, need to do well (imposed by others), feeling of incompetence.

Grading of Stress: Each item was answered in the form of score as 0 - no stress, 1- mild stress, 2moderate high stress, 3- High stress, 4- severe stress. Mild stress means it does not cause any or mild stress. Moderate stress indicates that it caused reasonable, but manageable stress. High stress indicates lot of stress and causes emotional disturbances and mildly compromises daily activities. Severe stress indicates severe emotional disturbances and compromise of daily activities.

The questionnaire was self-administered and distributed to the medical students. The students were instructed to adhere the instructions. Data was collected in the middle of academic period when there were no examinations, to avoid measurement bias.

\section{Data analysis}

The data were analysed using SPSS statistical analysis software v.20. The data were represented as mean \pm standard deviation (SD).

\section{RESULTS}

In this study, out of 250 students who attended the MSSQ-20, 109 (44\%) were males and 141(56\%) were females. Female preponderance was observed. The mean age of the students was found to be $22.17 \pm$ 0.94 years.

When evaluated for Academic related stressors (ARS), 138 students (55\%) showed severe stress, followed by high stress $74(30 \%)$, moderate stress $30(12 \%)$ and mild stress $8(3 \%)$. The data were shown in Table 1.

Table 1: Academic related stressors and its intensity

\begin{tabular}{|c|c|}
\hline Type of stress caused due to ARS & No of Students (\%) \\
\hline Mild & $8(3 \%)$ \\
\hline Moderate & $30(12 \%)$ \\
\hline High & $74(30 \%)$ \\
\hline Severe & $138(55 \%)$ \\
\hline
\end{tabular}

Evaluation for Intrapersonal and interpersonal development stressors (IRS) among the medical students reveals that $82(33 \%)$ were mild, $88(35 \%)$ were moderate, $56(22 \%)$ were severe, $24(10 \%)$ were high. The results were shown in Table 2.

Table 2: Intrapersonal and interpersonal development stressors and its intensity.

\begin{tabular}{|c|c|}
\hline Type of stress caused due to IRS & No of Students (\%) \\
\hline Mild & $82(33 \%)$ \\
\hline Moderate & $88(35 \%)$ \\
\hline High & $56(22 \%)$ \\
\hline Severe & $24(10 \%)$ \\
\hline
\end{tabular}

Teaching related stressors (TRS) evaluation among the students showed that, $88(35 \%)$ were mild, $79(32 \%)$ were moderate, $57(23 \%)$ were high and $26(10 \%)$ were high. The results were shown in table 3.

Table 3: Teaching related stressors and its intensity

\begin{tabular}{|c|c|}
\hline Type of stress caused due to TRS & No. of Students (\%) \\
\hline Mild & $88(35 \%)$ \\
\hline Moderate & $79(32 \%)$ \\
\hline High & $57(23 \%)$ \\
\hline Severe & $26(10 \%)$ \\
\hline
\end{tabular}

Social related stressors (SRS) evaluation among medical students displays that $82(33 \%)$ were mild, $104(42 \%)$ were moderate, $42(17 \%)$ were high and $22(8 \%)$ were severe. The results were shown in table 4.

Table 4: Social related stressors and its intensity

\begin{tabular}{|c|c|}
\hline Type of stress caused due to SRS & No of Students (\%) \\
\hline Mild & $82(33 \%)$ \\
\hline Moderate & $104(42 \%)$ \\
\hline High & $42(17 \%)$ \\
\hline Severe & $22(8 \%)$ \\
\hline
\end{tabular}


Desire related stressors (DRS) evaluation among the students reveals that $143(57 \%)$ were mild, $52(21 \%)$ were moderate, $31(12 \%)$ were high and $24(10 \%)$ were severe. The results were shown in table 5 .

Table 5: Desire related stressors and its intensity

\begin{tabular}{|c|c|}
\hline Type of stress caused due to DRS & No of Students (\%) \\
\hline Mild & $143(57 \%)$ \\
\hline Moderate & $52(21 \%)$ \\
\hline High & $31(12 \%)$ \\
\hline Severe & $24(10 \%)$ \\
\hline
\end{tabular}

Group activities related stressors (GARS) evaluation among the students shows that $44(18 \%)$ were mild, $77(31 \%)$ were moderate, $85(34 \%)$ were high and $44(17 \%)$ were severe. The data were shown in table 6.
Table 6: Group activities related stressors and its intensity

\begin{tabular}{|c|c|}
\hline Type of stress caused due to GARS & No of Students (\%) \\
\hline Mild & $44(18 \%)$ \\
\hline Moderate & $77(31 \%)$ \\
\hline High & $85(34 \%)$ \\
\hline Severe & $44(17 \%)$ \\
\hline
\end{tabular}

The mean score levels of various stressors domains were shown in Fig 1. Among them, ARS score was higher $(3.03 \pm 0.98)$ among the various domains. This was followed by GARS score $(2.27 \pm 0.91)$. The least score observed in DRS, $1.19 \pm 0.70$.

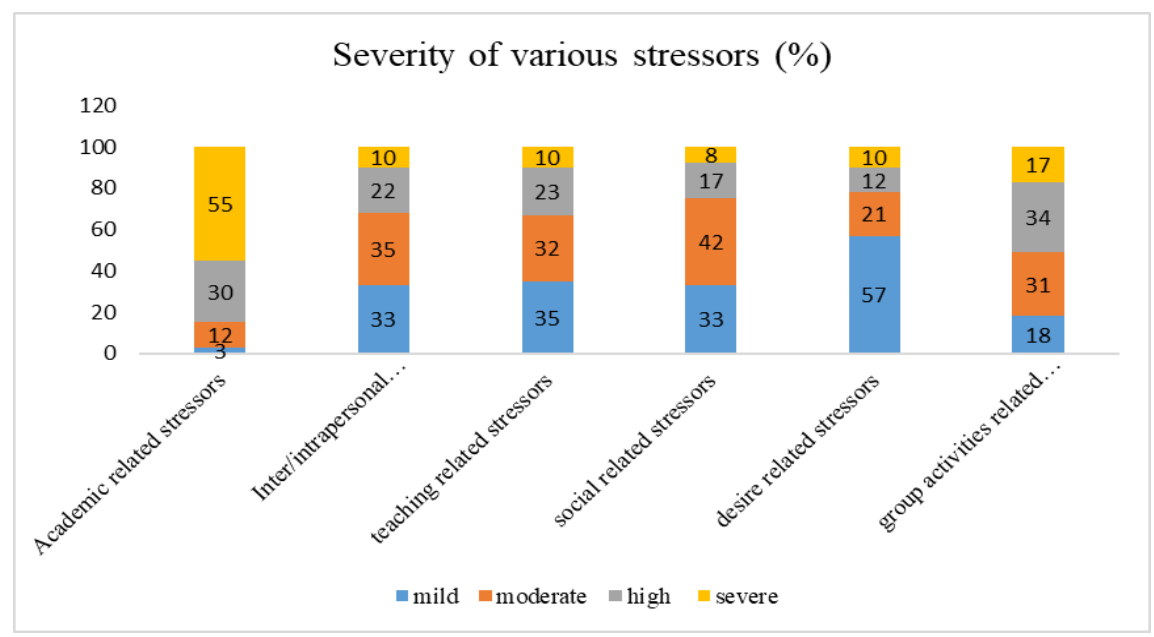

Fig. 1: Overview of the severity of various stressors

\section{DISCUSSION}

Medical students are prone to stressful situations during the course of their study (12-14). Long term exposure to stressor-scan result in altered emotional, physical and mental well-being of students. Further, excessive stress levels in medical students leads to decreased self-esteem, increased anxiety and depression, inability in solving personal conflicts, altered sleeping behaviour, increased substance use, decreased concentration and academic dishonesty (12). The present study has evaluated the stressors and measured the intensity of stress caused by the stressors among the final year medical students using Medical Students Stressor Questionnaire-20 items (MSSQ-20) rating scale in terms of academic related stressors (ARS), teaching related stressors (TLRS), intrapersonal and interpersonal development stressors (IRS), social related stressors (SRS), desire related stressors (DRS) and group activities related stressors (GARS) respectively (11).

ARS is related to the stress caused by the events related to university, college and educational institute. These events include grading and examination methods, strict academic schedule followed by the universities. The student-related stressors include less grades in examination, heavy course syllabus, lack of subject understanding, less time for revision, competition among the students and difficulty in answering during oral or written examinations. Elevated high score in this domain reflects that academic related events are the cardinal source of stress among the medical students. In the present study, majority of the students, $138(55 \%)$ displayed severe score of stress with respect to ARS domain. Previous reports show that high academic load and professional identity are the major stressful events experienced during undergraduate medical education (15). The academic load includes pressure related to achieve good grades in examination and clinical practice. Specifically, in the final year there is high pressure, since graduating and obtaining the degree is the main aim among the students. Further, preparing for postgraduate courses and choosing the right specialization also add further stress among the final year medical students (16).

IRS is the stress caused by the events related to the reaction between and within individuals. The events related to intrapersonal stressors includes lack of self-motivation, worry about personal life, home sickness and financial constraints. Meanwhile, interpersonal stressors are related to verbal, physical and emotional abuse caused by professors, personnel and staffs. In this study, $88(35 \%)$ of students displayed moderate score towards IRS. The reason 
might be due to the fact that the students understands that in the routine medical academia, expectations of the mentors may cause interpersonal and interpersonal related problems.

In this study, $88(35 \%)$ of students displayed mild form of TRS. The stress might be related to mounting clinical and theoretical task given by teachers to students, teachers feedback about the students, partiality or competency in teaching the students, lack of support from teachers and difficulty in understanding the lectures delivered by teachers. The strict behaviour of teachers is reported by various authors $(17,18)$. Further, medical students also claim that teachers lack in providing proper feedback and impose constant criticism rather than encouraging the students. Meanwhile, the unstructured teaching pattern along the patient's bedside, differences in teaching method also imposes stress among the students.

In this study, 104(42\%) of students are affected with moderate form of SRS. The SRS might be as a result of spending free time with friends and families, involving public activities, private time, interruption of work by others, listening to patient's problems. Thus, SRS indicates that medical students experience the difficulty in spending the time in activities related to social and communal (15).

In this study, 143(57\%) students are affected with mild form of DRS. It is generally related to the stress which involves forces that arise internally or externally and has significant impact on individual's emotions, attitude, thinking and behaviour. The important factors which cause DRS are lack of interest to study medicine, inappropriate course selection, pressure from parents and friends to take up the medicine course which reflects during the final year study course (1).

In this study, 85(34\%) students displayed high form of GARS. This stress arises as a result of group discussion participation, group presentations, expectations from the surrounding people to perform well. The high stress of GARS among the medical students in the present study is in corroboration with other published reports conducted in Malaysian medical schools $(19,20)$. Further, in a study done by Gupta et al. $70 \%$ of students reported the stress as a result of GARS (1).

Overall, the mean stress score level is higher in ARS, $3.03 \pm 0.98$ followed by GARS, $2.27 \pm 0.91$. This reflects that ARS and GARS are the major stressors responsible for stress among the final year medical students. Within the six major domains ARS and GARS occupies the major position in eliciting higher level of stress based on the stress score classification. Similar findings are reported in India and various medical schools globally $(21,22)$
Thus, it is vital to highlight that high stress among the students might decrease the mental stability, judgement and elevates the absenteeism. Overall, these effects hinder the academic ability of the students and restricts them in improving their skills. Further, high stress can lead to increased secretion of stress hormone cortisol and reduces the memory restoring functions of hippocampus and amygdala as a result of inhibition of glucocorticoid receptors (23). Meanwhile, it is the responsibility of the students to be aware the preventive methods for coping with stressful conditions. Further, it is responsible of the universities and medical schools to minimize the stressors by providing psychosocial and academic support methods to mitigate the students' stress.

\section{Study limitations}

The study faced few limitations. Generalizability of the study results is limited by the characteristic of the sample, which was recruited from a single private medical college. Despite assuring anonymity and confidentiality of their responses, under-reporting of diverse group of stressors could not be ruled out.

\section{CONCLUSION}

In the present study, the chronic stress was observed among the final year medical students. Academic Related Stressor and Group Activity Related Stressor are more prevalent, and it is at higher range among the students. Thus, substantial changes in academic structure to ameliorate the stress and introduction of stress management counselling programme is importantly needed to grow good inter and interpersonal ability among the students.

\section{CONFLICT OF INTEREST}

No potential conflict of interest was reported by the authors.

\section{REFERENCES}

1. Saravanan, C., Wilks, R. Medical students' experience of and reaction to stress: the role of depression and anxiety. Scientific World Journal. 2014; 2014: 737382.

2. Gupta, S., Choudhury, S., Das, M., Mondol, A., Pradhan, R. Factors Causing Stress Among Students of a Medical College in Kolkata, India. Educ Heal. 2015; 28(1): 92-95.

3. Hill, M. R., Goicochea, S., Merlo, L. J. In their own words: stressors facing medical students in the millennial generation. Med Educ Online. 2018; 23(1): 1530558.

4. Seedhom, A. E., Kamel, E. G., Mohammed, E. S., Raouf, N. R. Predictors of perceived stress among medical and nonmedical college students, Minia, Egypt. Int J Prev Med. 2019; 10: 107

5. Rotenstein, L. S., Ramos, M. A., Torre, M., Segal, J. B., Peluso, M. J., Guille, C., et al., Prevalence of Depression, Depressive Symptoms, and Suicidal Ideation Among Medical Students: A Systematic Review and Meta-Analysis. JAMA. 2016; 316(21): 2214-2236.

6. Chowdhury, R., Mukherjee, A., Mitra, K., Naskar, S., Karmakar, P. R., Lahiri, S. K. Perceived psychological stress among undergraduate medical students: Role of academic factors. Indian J Public Health. 2017;61(1):55-57. 
7. Singh, G., Hankins, M., Weinman, J. A. Does medical school cause health anxiety and worry in medical students? Med Educ. 2004; 38(5): 479-481.

8. Abdulghani, H. M., AlKanhal, A. A., Mahmoud, E. S., Ponnamperuma, G. G., Alfaris, E. A. Stress and its effects on medical students: a cross-sectional study at a college of medicine in Saudi Arabia. J Health PopulNutr. 2011; 29(5): 516-522.

9. Koochaki, G. M., Charkazi, A., Hasanzadeh, A., Saedani, M., Qorbani, M., Marjani, A. Prevalence of stress among Iranian medical students: a questionnaire survey. East Mediterr Health J. 2011; 17(7): 593-598.

10. Moutinho, I. L., Maddalena, N. C., Roland, R. K., Lucchetti, A. L, Tibiriçá, S. H., Ezequiel, O. D., et al., Depression, stress and anxiety in medical students: A cross-sectional comparison between students from different semesters. Rev Assoc Med Bras (1992). 2017; 63(1): 21-28.

11. Yusoff, M. S. B. A Multicenter Study on Validity of the Medical Student Stressor Questionnaire (MSSQ). Int Med J. 2011;18(1):14-18.

12. Dyrbye, L. N., Thomas, M. R., Shanafelt, T. D. Medical student distress: causes, consequences, and proposed solutions. Mayo Clin Proc. 2005; 80(12): 1613-1622.

13. Shah, M., Hasan, S., Malik, S., Sreeramareddy, C. T. Perceived stress, sources and severity of stress among medical undergraduates in a Pakistani medical school. BMC medical education. 2010 Dec; 10(1): 1-8.

14. Madhyastha, S., Latha, K. S., Kamath, A. Stress and coping among final year medicalstudents. AP J Psychol Med. 2014; 15(1): 74-80.

15. Shelke, U., Kunkulol, R., Narwane, S. Level of Stress in Final Year Mbbs Students at Rural Medical College: A Cross-Sectional Study. Int J Med Res Heal Sci. 2014; 3(4): 886-891.

16. Guruprakash, K. V., Mehta, S. G., Atul, B., Prakash, J., Divinakumar, K. J., Khan, S. A., et al., A study of relationship between perceived stress, coping pattern, burnout, and general psychopathology among the postgraduate medical students. Ind Psychiatry J. 2018; 27(1): 141-146.

17. Kohli, V., Dhaliwal, U. Medical students' perception of the educational environment in a medical college in India: A cross-sectional study using the Dundee Ready Education Environment questionnaire. J Educ Eval Health Prof. 2013; 10: 5.

18. Pai, P. G., Menezes, V., Srikanth., Subramanian, A. M., Shenoy, J. P. Medical student's perception of their educational environment. J Clin Diagn Res. 2014;8 (1): 103-107.

19. Yusoff, M. S., Abdul Rahim, A. F., Yaacob, M. J. Prevalence and Sources of Stress among Universiti Sains Malaysia Medical Students. Malays J Med Sci. 2010; 17(1): 30-37

20. Elias, H., Ping, W. S., Abdullah, M. C. Stress and academic achievement among undergraduate students in Universiti Putra Malaysia. Procedia-Social and Behavioral Sciences. 2011; 29: 646-655.

21. Anuradha, R., Dutta, R., Raja, J. D., Sivaprakasam, P., Patil, A. B. Stress and Stressors among Medical Undergraduate Students: A Cross-sectional Study in a Private Medical College in Tamil Nadu. Indian J Community Med. 2017. Oct-Dec; 42(4): 222-225.

22. Rahman, A. A., Al Hashim, B., Alhiji, N. K., Al-Abbad, Z. Stress among medical saudi students at College of Medicine, King Faisal University. Journal of Preventive Medicine and Hygiene. 2013; 54(4): 195-199.

23. Conrad, D. C. Chronic Stress-Induced Hippocampal Vulnerability: The Glucocorticoid Vulnerability Hypothesis. Rev Neurosci. 2008; 19(6): 395-411. 